\title{
DESIGN AND DEVELOPMENT OF 3-STAGE DETERMINATION OF DAMAGE LOCATION USING MAMDANI-ADAPTIVE GENETIC-SUGENO MODEL
}

\author{
SASMita SAHU \\ School of Mechanical Engineering, Kalinga Institute of Industrial Technology, KIIT University, Bhubaneswar, Odisha, \\ India; e-mail: gudusasmita@gmail.com \\ Priyadarshi Biplab Kumar, Dayal R. Parhi \\ Department of Mechanical Engineering, National Institute of Technology, Rourkela, Odisha, India \\ e-mail: p.biplabkumar@gmail.com; dayalparhi@yahoo.com
}

\begin{abstract}
Damage detection in structural elements like beams is one of important research areas for health monitoring. Initiation of a fault in the form of a crack or any damage puts a limitation on the service life of a structural member. So, in this paper, a method is proposed which uses the advantages of soft computing techniques like Fuzzy Inference Systems (Mamdani and Sugeno) and Adaptive Genetic Algorithm for three stage refinement of the data base generated using dynamic responses from a cracked fixed-free aluminum alloy beam element. For the crack element reference, a finite element model of a single transverse crack has been considered. The proposed method describes both Mamdani and Sugeno Fuzzy Inference Systems for training of damage parameters. In the Adaptive Genetic Algorithm, a statistics based method has been incorporated to limit the randomness of the search process. Finally, the results from the Mamdani-Adaptive Genetic-Sugeno model (MAS) are validated with the results from the experimental analysis.
\end{abstract}

Keywords: damage, Mamdani FIS, Sugeno FIS, Adaptive Genetic Algorithm, vibration, natural frequencies

\section{Introduction}

Cracks in structural and machine members indicate the amount of serviceability of the structures. Crack initiation is obvious in most of engineering structures due to environmental and working conditions. Usually, a hairline crack is visible and can be inspected using a crack gauge, fiber optical sensor or laser sensor. But, it becomes very difficult to detect a very small crack whose location is very dangerous for the structural element (Jaiswal and Pande, 2015). Cracks change dynamic responses of structures like natural frequencies and mode shapes (Pawar et al., 2007). These changes in the dynamic properties can be used to detect the presence of the crack (Fegade et al., 2014). So, the vibration based methods are gently getting popularized for crack detection. These methods have the ability of convenience measurement by collecting the variation in modal properties (Waghulde and Kumar, 2014). The analytical methods used for damage detection cost time and are subjected to human error. With the invention and development of finite element analysis, many of the researchers are using this method for the analysis of dynamics of cracked beams (Ranjbaran and Ranjbaran, 2013). Though finite element analysis is an approximation method, it can give great data with less time consumption. So, this paper uses finite element analysis for modeling of the crack. Yuan et al. (2014) have recently proposed one of the methods to make re-meshing easier for analyzing crack propagation area by incorporating a radial point interpolation method. The artificial intelligence techniques with vibration based methods can make a powerful tool for online detection of the damage. These techniques can learn offline 
vibration signatures to evaluate the condition monitoring status of large complex structures both in static and dynamic conditions.

In this research work, a knowledge based computational method and an evolutionary working on the "Natural Selection" is addressed for vibration analysis of the cracked structural element. From the invention of this logic, it has been successfully applied to different research fields. Many researchers have also used Fuzzy Logic and its advance versions in the field of damage detection and localization (Verma et al., 2013). From the vast literature available, it can be observed that most of the researchers have used Mamdani fuzzy inference system for their analyses. Chandrashekhar and Ganguli (2009) proposed one of the analytical methods for modal analysis using Modal Curvature and Fuzzy Logic. Zhu and Wu (2014) depicted a method for rapid structural damage detection using ANFIS and interval modeling technique, in which the ANFIS model has been designed using Sugeno FIS.

In the first Section of the paper, both Mamdani and Sugeno FIS are described for crack detection. Here, Fuzzy Logic has been used to learn the dynamics of the cracked structure for damage detection. Two types of membership functions have been proposed to design variables of the current problem. In the second part of the paper, an Adaptive Genetic Algorithm method has been narrated. The main notion of this adaptive search algorithm is to simulate the process necessary for evolution (Shahidi et al., 2015). This stochastic search algorithm provides an intelligent exploitation of search space to solve a problem. Khaji and Mehrjoo (2014) proposed a new method using Genetic Algorithm for the determination of the crack locations of an arbitrary number of transverse cracks. The Search Space in GA plays an important role in finding the absolute solution as; Genetic Algorithms (GAs) are random search algorithms. These algorithms can be applied to many real world problems of complex and intricate nature which are tough to be solved by traditional or conventional methods. Various statistical methods are also incorporated in damage detection problems to control the randomness and nonlinearity of the damage indices which are trained in different techniques (Niezrecki, 2015). Therefore, in this paper, Regression Analysis has been incorporated for the data analysis of the problem, which makes the problem more adaptive (Dervilis et al., 2015). Finally, in the third part, the data base generated from the finite element analysis and experimental analysis is trained in the proposed method using the 3-stage Mamdani-Adaptive Genetic-Sugeno model.

The first three natural frequencies are extracted from FEA and converted into relative values. The relative values of the natural frequencies (rff, rsf, rtf) are found out by comparing the natural frequencies of the uncracked beam and the cracked beam. Relative values of the crack depth and crack location $(r c d, r c l)$ are also found out using the similar method. This work considers only the natural frequencies because they are less prone to error while calculating. The relative first natural frequency $(r f f)$, relative second natural frequency $(r s f)$ and relative third natural frequency $(r t f)$ are treated as the input variables in the proposed method. The outputs from the system are the relative values of the crack depth $(\mathrm{rcd})$ and crack location $(\mathrm{rcl})$ which, in turn, contains the information of damage severity. The reason behind the idea to take the relative values of the input and output variables is to lessen the coding error and running time of the algorithm when fed to it. The proposed method is a type of inverse analysis problem. Here, by giving the crack depths and crack locations, the natural frequencies are found out using theoretical, finite element and experimental analyses. These set of crack locations and first three natural frequencies are used as the data set.

\section{Finite element analysis approach for cracked beam element}

The Euler-Bernoulli beam model is assumed for the finite element formulation. The crack in this particular case is assumed to be an open crack, and the damping is not being considered in this 
theory. The geometry of the cracked beam is described in Fig. $1\left(P_{1}-\right.$ axial load, $P_{2}-$ bending moment, $L_{1}$ - distance of the crack from the free end of the beam, $L$ - length of the cantilever beam, $B$ - breadth of the cantilever beam, $W$ - width of the cantilever beam, $a_{1}$ - crack depth.

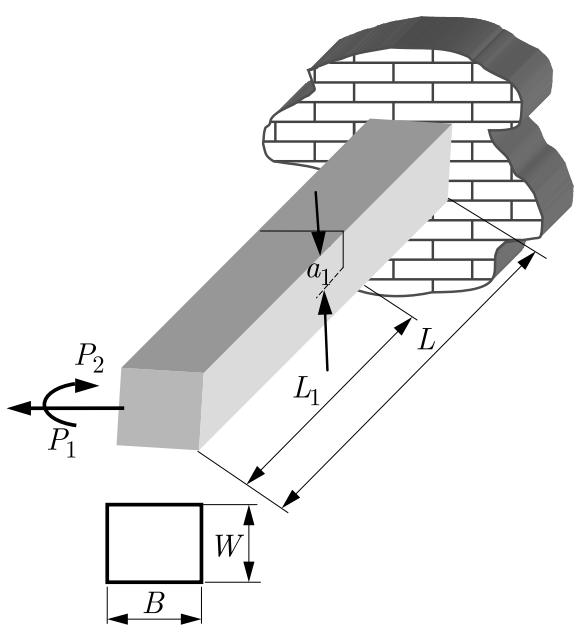

Fig. 1. Geometry of the cracked cantilever beam

The free bending vibration of a Euler-Bernoulli beam of a constant rectangular cross section is given by the following differential equation

$$
E I \frac{d^{4} y}{d x^{4}}-m \omega_{i}^{2} y=0
$$

where $m$ is the mass of the beam per unit length $[\mathrm{kg} / \mathrm{m}], \omega_{i}$ - natural frequency of the $i$-th mode $[\mathrm{rad} / \mathrm{s}], E-$ modulus of elasticity $\left[\mathrm{N} / \mathrm{m}^{2}\right]$ and $I$ is the moment of inertia $\left[\mathrm{m}^{4}\right]$. By defining $\lambda^{4}=m \omega_{i}^{2} /(E I)$, the equation is rearranged as a fourth-order differential equation as follows

$$
\frac{d^{4} y}{d x^{4}}-\lambda^{4} y=0
$$

A general solution to the equation is

$$
y=A \cos \lambda_{i} x+B \sin \lambda_{i} x+C \cosh \lambda_{i} x+D \sinh \lambda_{i} x
$$

where $A, B, C, D$ are constants and $\lambda_{i}$ is a frequency parameter.

The governing differential equation for the system is given as

$$
\mathbf{M} \ddot{\mathbf{x}}+\mathbf{C} \dot{\mathbf{x}}+\mathbf{K} \mathbf{x}=\mathbf{F} \sin (\omega t)
$$

where $\mathbf{M}, \mathbf{K}, \mathbf{C}$ is the mass, stiffness and damping matrix, respectively, $\mathbf{F}$ is the external force.

But, it is assumed that there is no damping and there is no external force applied to the system. So, the governing equation becomes

$$
\mathbf{M x}+\mathbf{K x}=\mathbf{0}
$$

The equation of motion for natural frequencies for undamped free vibration is given in equation (2.5). To solve equation (2.5), it is assumed that

$$
\mathbf{x}=\phi \sin (\omega t)
$$

where $\phi$ is the eigenvector or mode shape, $\omega$ - circular natural frequency. 
Substituting the differential equation of the assumed solution into Eq. (2.5), the equation of motion will be changed to

$$
\omega^{2} \mathbf{M} \phi \sin (\omega t)+\mathbf{K} \phi \sin (\omega t)=\mathbf{0}
$$

After simplification, it becomes

$$
\left(\mathbf{K}-\omega^{2} \mathbf{M}\right) \phi=\mathbf{0}
$$

The equation is called the eigenequation. The basic form of the eigenvalue problem is

$$
(\mathbf{A}-\lambda \mathbf{I}) \mathbf{x}=\mathbf{0}
$$

where $\mathbf{A}$ is the square matrix, $\lambda$ - eigenvalues, $\mathbf{I}$ - identity matrix, $\mathbf{x}$ - eigenvector.

In structural analysis, the eigenequation is written in terms of $\mathbf{K}, \mathbf{M}$, and $\omega$ with $\omega^{2}=\lambda$.

There are two possible solutions to equation (2.8)

1. If $\left|\left(\mathbf{K}-\omega^{2} \mathbf{M}\right) \phi\right| \neq 0$ is a trivial solution where $\phi=\mathbf{0}$

2. If $\left(\mathbf{K}-\omega^{2} \mathbf{M}\right) \phi=\mathbf{0}$ is a non-trivial solution where $\boldsymbol{\phi} \neq \mathbf{0}$

$$
\left|\left(\mathbf{K}-\omega^{2} \mathbf{M}\right)\right|=0 \quad|(\mathbf{K}-\lambda \mathbf{M})|=0
$$

The determinant is zero only for discrete eigenvalues

$$
\left|\left(\mathbf{K}-\omega^{2} \mathbf{M}\right)\right| \phi_{i}=0 \quad i=1,2,3, \ldots
$$

Adopting Hermitian shape functions, the stiffness matrix of the two-noded beam element without a crack is obtained using the standard integration based on the variation in flexural rigidity

$$
\mathbf{K}_{c}^{e}=\mathbf{K}^{e}-\mathbf{K}_{c}
$$

Here, $\mathbf{K}_{c}^{e}$ is the stiffness matrix of the cracked element, $\mathbf{K}^{e}-$ element stiffness matrix, $\mathbf{K}_{c}-$ reduction in the stiffness matrix due to the crack.

According to (Peng et al., 2007), the matrix $\mathbf{K}_{c}$ is

$$
\mathbf{K}_{c}=\left[\begin{array}{cccc}
K_{11} & K_{12} & -K_{11} & K_{14} \\
K_{12} & K_{22} & -K_{12} & K_{24} \\
-K_{11} & -K_{12} & K_{11} & -K_{14} \\
K_{14} & K_{24} & -K_{14} & K_{44}
\end{array}\right]
$$

It is assumed that the crack does not affect the mass distribution of the beam. The consistent mass matrix of the beam element is given as

$$
\mathbf{M}^{e}=\int_{0}^{1} \rho \mathbf{A} \mathbf{H}^{\mathrm{T}}(x) \mathbf{H}(x) d x \quad \mathbf{M}^{e}=\frac{\rho}{20} \mathbf{A} \mathbf{I}\left[\begin{array}{cccc}
156 & 221 & 54 & -131 \\
221 & 41^{2} & 131 & -31^{2} \\
54 & 131 & 156 & -221 \\
-131 & -31^{2} & -221 & 41^{2}
\end{array}\right]
$$

The natural frequency then can be calculated from relation (2.11).

\section{Fuzzy logic approach for structural damage detection}

Fuzzy logic can operate on imprecise, noisy inputs, but the output is a very smooth unit. It incorporates a simple rule based approach to solve control problems rather than solving it mathematically. The fuzzy logic controller model is empirically based on the designer's experience rather than the technical understanding of the system. In this work, the natural frequencies from the damaged beam element are used as the input to the fuzzy controller as shown in Fig. 2, and the outputs from the fuzzy controller are the crack depth and crack location. 


\subsection{Fuzzy inference systems}

Fuzzy Inference Systems (FIS) are of two types, i.e., Mamdani FIS and Takagi-Sugeno FIS. In this work, the Mamdani FIS consists of the following steps. Figure 2 describes a fuzzy controller using shuffled membership functions.

1. Fuzzification (use of the membership function for graphical presentation of the database)

2. Rule evaluation (implementation of the rules)

3. Defuzzification (obtaining the crisp values of the results)

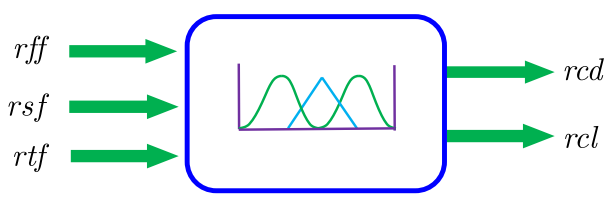

Fig. 2. Fuzzy logic controller using input and output membership functions

\subsubsection{Mamdani fuzzy inference system for crack detection}

The fuzzy logic system consisting of the Mamdani fuzzy inference model is implemented through the following steps.

1) Fuzzification: First, the crisp input and output variables are fuzzified using fuzzy sets. The degrees of membership of these variables are defined.

2) Rule aggregation and evaluation: The fuzzified variables are used to form fuzzy rules. If a fuzzy rule consists of many antecedent parts, they are joined by fuzzy operators (AND or NOT). For the Mamdani fuzzy inference, the $i$-th rule can be mathematically expressed as

$$
R_{i}: \quad \Omega x_{1}=A_{i 1} \wedge x_{2}=A_{i 2} \wedge \ldots \wedge x_{n}=A_{i n} \Rightarrow y_{1}=B_{i 1} \wedge y_{2}=B_{i 2}
$$

where $A_{i 1}, A_{i 2}, \ldots, A_{i n}$ and $B_{i 1}, B_{i 2}$ are the fuzzy sets.

The membership values of all rule consequents are combined into a single fuzzy set.

3) Defuzzification: The most common type of the defuzzification method is the centroid method. This defuzzifier method has been used in all the fuzzy logic system of this research work. This method finds a point representing the COG (centre of gravity) of the aggregated fuzzy set $A$ in the interval $[a, b]$. The following figure shows the Mamdani FIS applied in this work.

The other types of defuzzification methods are the mean of maximum, weighted average method, height method, etc.

\subsubsection{Sugeno fuzzy inference system for crack detection}

Steps used in the Takagi-Sugeno fuzzy inference modeling for damage detection are as follows.

1. General structure of the Sugeno fuzzy inference begins with fuzzification of the input ( rff, $r s f, r t f)$ and output variables $(r c d, r c l)$ within the defined range using different types of membership functions (triangular, trapezoidal, Gaussian, etc.) the like Mamdani FIS.

For the current problem, let $x_{1}, x_{2}, x_{3}$ be input variables defined on the reference sets $X_{1}, X_{2}, X_{3}$ and let $y_{1}$ and $y_{2}$ be output variable defined on the reference sets $Y_{1}$ and $Y_{2}$. 
Then FIS has three input variables and two output variables. Further, each set $X_{j}$, $j=1,2,3$, can be divided into $i=1,2, \ldots, n$ fuzzy sets

$$
\mu_{j, 1}(x), \mu_{j, 2}(x), \ldots, \mu_{j, i}(x), \ldots, \mu_{j, n}(x)
$$

where $\mu$ is the membership function value.

2. The next step comprises of the formation of the fuzzy base rules. The $l$-th if-then rule $R_{k}$ in Sugeno FIS can be written in the following form

$$
\begin{gathered}
R_{l}: \quad \Omega x_{1}=L_{1, i(1, l)} \wedge x_{2}=L_{2, i(1, l)} \wedge x_{3}=L_{3, i(1, l)} \\
\Rightarrow y_{1}=f_{1}\left(x_{1}, x_{2}, x_{3}\right) \wedge y_{2}=f_{2}\left(x_{1}, x_{2}, x_{3}\right)
\end{gathered}
$$

where $L_{1, i(1, l)}, L_{2, i(1, l)}, L_{3, i(1, l)}$ are the linguistic variables.

$y_{1}=f_{1}\left(x_{1}, x_{2}, x_{3}\right), y_{2}=f_{2}\left(x_{1}, x_{2}, x_{3}\right)$ are linear polynomial functions, $l=1,2, \ldots, N$

$$
R_{l}: \quad \Omega x_{1}=L_{1, i(1, l)} \wedge x_{2}=L_{2, i(1, l)} \wedge x_{3}=L_{3, i(1, l)} \Rightarrow y_{1}=k_{1} \wedge y_{2}=k_{2}
$$

where $k_{1}, k_{2}$ are constants. Equation (3.4) describes the zero order Sugeno FIS rule.

3. The third step describes the defuzzification procedure in the Sugeno FIS. The output weight of $y_{l}$ of each of the $l$-th if-then rule $R_{l}$ is aggregated by

$$
w_{l}=\mu\left(x_{1}\right) \wedge \mu\left(x_{2}\right) \wedge \mu\left(x_{3}\right)
$$

The final output after aggregation of $N$ rules is computed as

$$
y=\frac{\sum_{l=1}^{N} y_{l} w_{l}}{\sum_{l=1}^{N} w_{l}}
$$

\section{Adaptive genetic algorithm for damage detection}

Genetic Algorithms (GAs) are based on the evolution of natural selection and genetics. This algorithm was first developed by Holland (1992). This is a heuristic search algorithm. The main notion of this adaptive search algorithm is to simulate the process necessary for evolution. This stochastic search algorithm provides an intelligent exploitation of search space to solve a problem. The search space in GA plays an important role in finding the absolute solution as; genetic algorithms (GAs) are random search algorithms. Although GAs can be used to find solutions to very complicated real world problems, they are very much simple to use and understand. It is able to search through a variety and huge combination of parameters to find the best match. But, sometimes, the GA becomes unidirectional without expediting the entire search space. The accessibility to the better solution in the search space becomes easier if there exists a relationship between the independent and dependent variables in a problem. Therefore, in this work, regression analysis has been incorporated for the data analysis of the problem, which makes the problem more adaptive.

The operators and procedure or the natural evolution process is similar to the simple genetic algorithm. The domain containing viable solutions is called the search space. Each individual point in the search space is a feasible solution. These solutions are ranked by their fitness values for selection. The important features of the algorithm are the genetic operators. These operators try to imitate the process of natural selection of the evolution process. The genetic operators, 
crossover and mutation perform two different roles. Crossover tries to direct the population towards a local solution which leads to premature convergence of the algorithm. But, mutation is a divergence operation that tries to introduce diversity in the population, so that there will be more exploitation of the search space. This paves the way towards achieving a better solution. But the mutation amount in every generation is kept small and should affect a few members of a population. Otherwise, the entire solution space will be changed, and the algorithm will become directionless.

\subsection{Regression analysis for the generation of the data pool for GA}

Regression analysis comes under the category of supervised learning based on the statistical modeling of the problem. Regression analysis is used to find the relationship between two variables. This method is mainly a statistics based method. It can be described in the form of cause (independent variable) and effect (dependent variable). Several researchers have used regression analysis for the data base analysis of damage detection problems, but no one has combined it with genetic algorithms.

This analysis mainly related to dynamic responses of a cracked structural element with a damage extent. It is often realized that damage parameters are often scalar values, so univariate statistical tests can be utilized to find out possible changes among parameter vectors associated with a definite location. It is also expected that the computational cost of such statistical testing is less than that of the parameter extraction. The present problem is a case of multiple regression analysis due to the presence of multiple input and output variables.

For the present analysis, $Y=r f f, r s f$, $r t f$ and $X=r c d, r c l$.

The linear equations for the current problem relating the dependent and independent variables are as in the following

$$
\begin{aligned}
& Y_{1}=p_{1}+q_{1}\left(X_{1}\right)+q_{2}\left(X_{2}\right)+q_{3}\left(X_{3}\right) \\
& Y_{2}=p_{1}+q_{4}\left(X_{1}\right)+q_{5}\left(X_{2}\right)+q_{6}\left(X_{3}\right)
\end{aligned}
$$

For the data extraction using the regression analysis method, direct values of the variables are used. After analysis, the values of the data are converted to the relative values.

\subsection{Implementation of adaptive genetic algorithm for fault detection in cracked structures}

As we know Genetic Algorithm (GA) is a search algorithm, it can be well applied to the current problem. In this problem, it is required to find the relative crack depth and relative crack location to a corresponding set of relative natural frequencies from the field signals. For this, first, a database is prepared from the results of theoretical, finite element and experimental analyses. Then rest of the steps are done according to the genetic algorithm. For all types of evolutionary algorithms, the encoding is a must. The advancement of the algorithm and the application of the operators mainly depend on the representation scheme adapted for the algorithm.

The encoding of chromosomes used in the current problem is the bit string/binary encoding. Each gene of the chromosome may be either 0 or 1 . The gene/bit strings contain the information about the solution. In this approach, each chromosome contains five genes (rff, rsf, rtf, rcd and $r(\mathrm{l})$. Each gene contains four bits, so each chromosome contains twenty bits. After the assignment of the representation scheme to the chromosomes, the problem proceeds towards the algorithm.

Following are the steps used in the genetic algorithm.

1) First of all, the variables and fitness functions are selected.

The GA begins by defining input variables whose values are to be optimized using the fitness function and output variables whose values are to be anticipated using genetic operators. 
The fitness function to be minimized is defined as

$$
\text { Fitness Function }=\sqrt{\left(r f f_{f d}-r f f_{x_{1}, i}\right)^{2}+\left(r s f_{f d}-r s f_{x_{1}, i}\right)^{2}+\left(r t f_{f d}-r t f_{x_{1}, i}\right)^{2}}
$$

where $r f f_{f d}$ is the first natural frequency of the field, $r f f_{x}$ - relative first natural frequency, $r s f_{f d}$ - second natural frequency of the field, $r s f_{x}$ - relative second natural frequency, $r t f_{f d}$ - third natural frequency of the field, $r t f_{x}$ - relative third natural frequency, $i$ - number of iterations.

The less the difference between the field and random frequency, the higher rank will be acquired by chromosome.

2) A data pool (initial population) containing ten numbers of data sets (individuals) is created. This data pool is acquired from FEA and theoretical analysis.

3) Two parents (i.e., two data set) from data pool (i.e., from ten data sets) using the fitness function are selected.

4) The selected parents undergo crossover. Here, two-point crossovers are used. As the chromosomes contain five parameters, and each parameter contains four bits, so the chromosome contains twenty bits. The crossover points are chosen four bits left of the chromosome and four bits right of the chromosome. Figure 3 shows the presentation of the parent with crossover points.

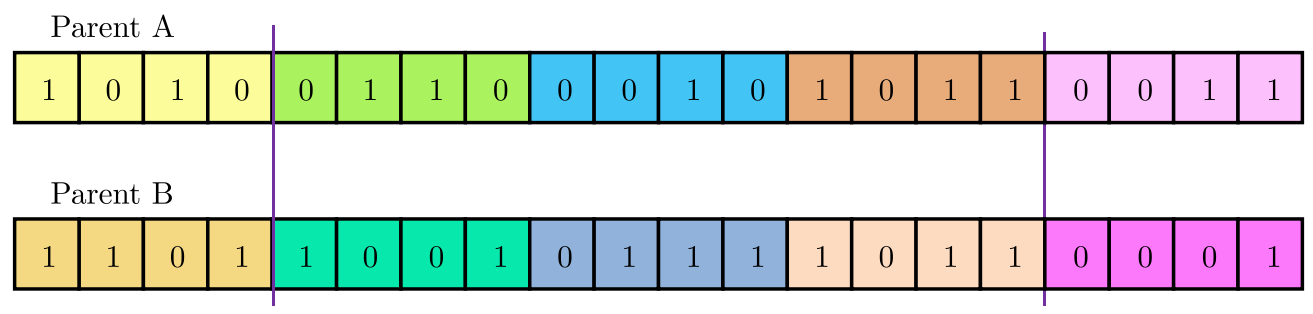

Fig. 3. Parent chromosomes with crossover points

5) The children (two numbers) from the parents are found out. Figure 4 describes the application of two-point crossover points in the genetic algorithm for damage detection.

6) After crossover, mutation is performed. In the proposed paper, as we have used binary encoding, the mutation rate used is $0.1 \%$ of the string. As the chromosome consists of 20 bits, only two bits at a time are flipped or altered. Figure 5 describes the application of the tossing type of mutation in the genetic algorithm for damage detection.

7) Again, fitness evaluation of the parents and the offspring is done. Then, the fitness values of the parents and children are compared to find out the best fit member.

8) If the child comes as the best fit, then it is added to the data pool, and a new set of the data pool is created. If a parent comes as the best fit, then the desired output $(r c d, r c l)$, is the output belonging to that set.

9) Steps from 2-8 are repeated in each iteration till the algorithm meets the threshold values.

The algorithm terminates when it meets the threshold values. The threshold values for GA to stop are as below. The algorithm stops when it meets any of the criteria first.

i. 50 generations

ii. Maximum time elapsed (running time of the algorithm, i.e., two minutes) 


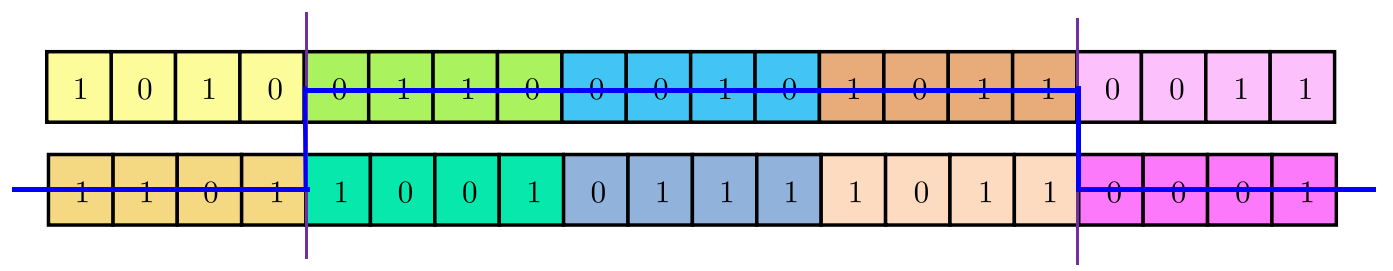

Child A
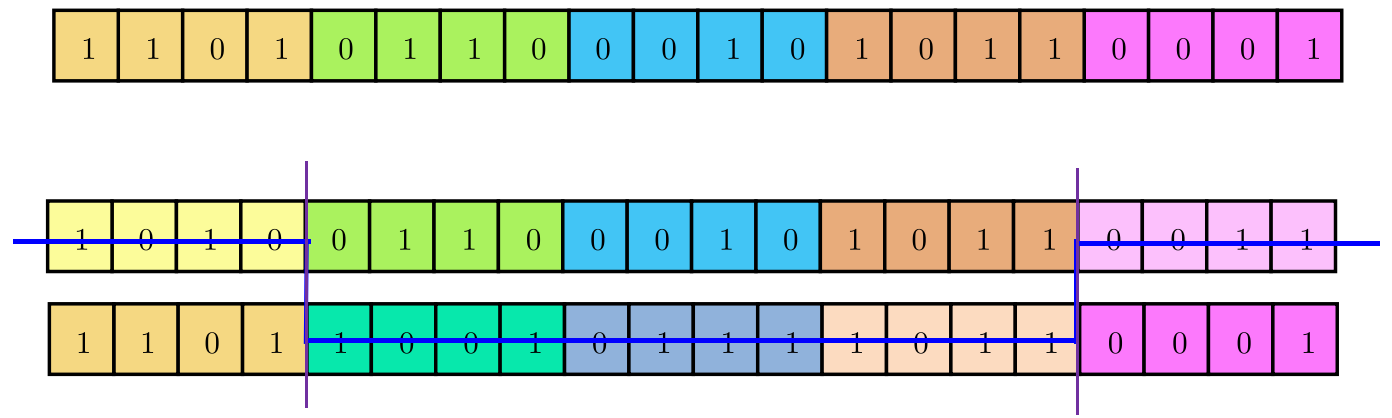

Child B

\begin{tabular}{|l|l|l|l|l|l|l|l|l|l|l|l|l|l|l|l|l|l|l|l|}
\hline 1 & 0 & 1 & 0 & 1 & 0 & 0 & 1 & 0 & 1 & 1 & 1 & 1 & 0 & 1 & 1 & 0 & 0 & 1 & 1 \\
\hline
\end{tabular}

Fig. 4. Two-point crossover used for producing the off-springs

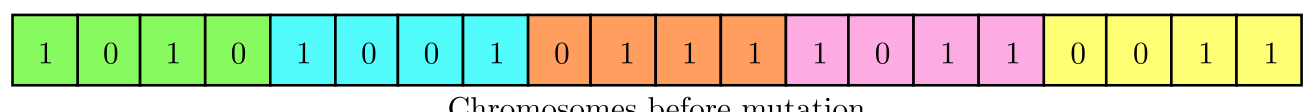

Chromosomes before mutation

\begin{tabular}{|l|l|l|l|l|l|l|l|l|l|l|l|l|l|l|l|l|l|l|l|}
\hline 1 & 1 & 1 & 0 & 1 & 0 & 0 & 1 & 0 & 1 & 1 & 1 & 1 & 0 & 1 & 1 & 0 & 1 & 1 & 1 \\
\hline
\end{tabular}

Fig. 5. Description of the mutation process implemented in damage detection

\section{Analysis of 3-stage determination of damage location using Mamdani-Adaptive Genetic-Sugeno model}

From the vast literature available in the structural damage detection using Fuzzy Logic and Genetic Algorithm for following conclusions have been drawn.

Several authors have used fuzzy logic for damage detection using the Mamdani FIS. The membership functions used in the Mamdani FIS are also different. Some have used simple membership functions (Saridakis et al., 2008) while others have used combinations of membership functions (Parhi et al., 2011; Thatoi et al., 2014). It has been noticed by the researchers that the Mamdani FIS using a combination of membership functions gives better results than the simple membership functions. Fewer researchers have used the Sugeno model for damage detection. Most of the researchers have used Sugeno model with an artificial neural network for damage detection. When the results of the Mamdani FIS and Sugeno FIS are compared, it has been observed that the Sugeno FIS gives better results than the Mamdani FIS. Likewise, many researchers have used genetic algorithms (Vakil-Baghmisheh et al., 2008) for damage detection. A simple Genetic Algorithm is very easy to understand and simple to apply, once the designer has understood the dynamics of the problem. Though it is very widely used in many of engineering fields, it has some shortcomings like attainment of global solution (optimization). This occurs mainly due to the random search of the solution space which is also time consuming. So, in the proposed adaptive genetic algorithm, regression analysis has been incorporated to the statistical modeling of the variable relationship, so that the genetic algorithm will take less time to cover the entire solution space. 
After analyzing all the above stated factors, the current method is proposed. In the proposed method, 3 -stage treatment of the data generated is done.

\subsection{Design and development of 3-stage determination of damage location using the Mamdani-Adaptive Genetic-Sugeno (MAS) model}

The previous Sections of this paper have discussed the benefits of the Mamdani FIS, Sugeno FIS and the adaptive genetic algorithm. So, in this Section, a method comprising 3-stage treatment of the data is used for damage detection in beams with two end conditions (fixed-free, fixed-fixed). The data pool which is to be treated in multiple stages, finally to give the crack location $(r c d, r c l)$, is generated from the theoretical, finite element analysis and experimental analysis.

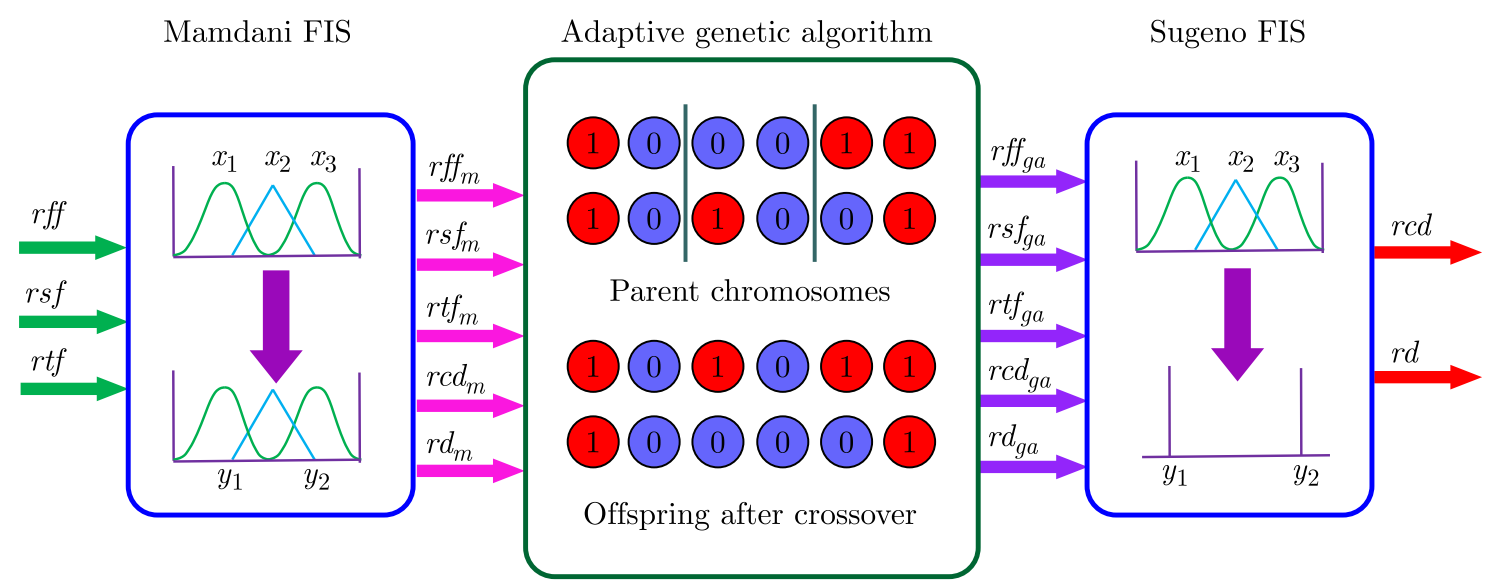

Fig. 6. Presentation of 3-stage determination of damage location using the Mamdani-Adaptive Genetic-Sugeno (MAS) model

In the first stage, the data pool is treated in the fuzzy Mamdani FIS. Here, the Mamdani FIS has been designed using shuffled membership functions comprising of triangular and Gaussian MFs. The efficiency of the Mamdani FIS using shuffled membership functions has already been noticed in Section 3 of this paper. Also from the works of other researchers, it has been observed that a combination of membership functions gives better results than the simple membership functions. In the Mamdani FIS, by changing the input values, the output values are obtained for at least hundred runs. After the training of the data pool in the Mamdani segment, the transit data is generated with the crack location as $r c d \_m$ and $r c l \_m$. After obtaining the first set of data from the Mamdani FIS; it is treated in the adaptive genetic algorithm segment as described in Section 4 of this paper.

During the treatment of the data in this segment, the results get closer towards the global solution due to the treatment of the data in regression analysis. After applying regression analysis to the hundred data sets, half of the data sets are taken to be trained in the genetic algorithm. The crack locations from the genetic algorithm are named as $r c d \_g a$ and $r c l \_g a$. The GA is then run for hundred times. Out of the hundred runs, fifty different data sets are taken to make the second data pool.

The second data pool is then trained in the Sugeno FIS after the second stage treatment of the data pool; the data gets more refined. After the training of the second data pool in the Sugeno FIS, the final results of the 3-stage training of the data are obtained with better crack location parameters. Due to the achievement of better results from the Sugeno FIS, it has been kept in the last segment of the training procedure after the adaptive genetic algorithm segment. Figure 6 depicts a pictorial presentation of the stages of the 3 -stage determination of damage location using the Mamdani-Adaptive Genetic-Sugeno model. 


\section{Experimental set-up used in the fault detection of a cracked beam}

The instruments used in the free vibration analysis of the fixed-free beam are an impact hammer, vibration pick-up, vibration analyzer and vibration indicator (Fig. 7). Using the impact hammer, the cracked fixed-free beam is excited in the free vibration mode. The vibration analyzer is PULSE LAB Prolite 3560. The excitation parameters are picked up by the vibration pick-up or an accelerometer. Then, these parameters are fed to the vibration analyzer, where the parameters are analysed and the results are shown in the vibration indicator.
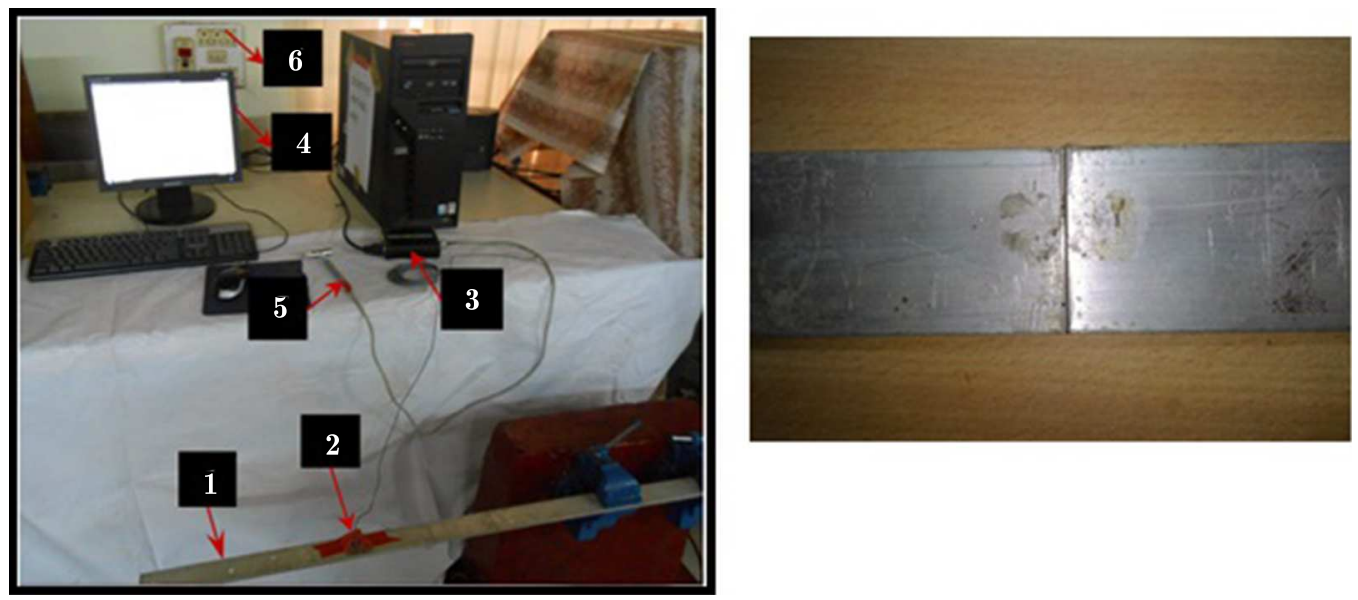

Fig. 7. Experimental set-up with the cracked beam; 1 - cracked fixed-fixed beam with a single crack, 2 - vibration pick-up, 3 - vibration analyzer, 4 - vibration indicator, 5 - impact hammer,

6 - power distribution

Several tests have been conducted using the experimental setup on aluminium alloy beam specimens $(800 \mathrm{~mm} \times 38 \mathrm{~mm} \times 8 \mathrm{~mm})$ with a transverse crack for determining the natural frequencies at different crack locations and crack depths. These specimens are given vibration by impact hammer, and the 1st, 2nd and 3rd natural frequencies are recorded in the vibration indicator.

\section{Result and discussions}

The percentage error is calculated using the following formula

$[($ FEA result - result from the proposed technique)/(FEA result) $] \cdot 100$

$[($ Exp. result - result from the proposed technique $) /($ Exp. result $)] \cdot 100$

Total error in $\%=(\%$ error in $r c d+\%$ error in $r c l) / 2$

The present work describes the damage detection method for cracked structural elements. First, the transverse hairline crack has been modeled using the finite element method. In the finite element analysis, the cracked structure has been modeled assuming the Euler-Bernoulli beam model. Then, the vibration parameters which are the indices of damage are extracted from the finite element analysis. Then, a data base consisting of the cause (crack depth, crack location) and effect (change in first three natural frequencies) of initiation of the crack is made. Then, the data base is trained in different computational techniques (fuzzy logic (Mamdani FIS and Sugeno FIS), adaptive genetic algorithm and Mamdani-Adaptive Genetic-Sugeno model) to find the crack location.

Though with the invention of soft computing methods have made it easier to find good results at the fingertip by just running a program, finding a global optimization method remains a major challenge in engineering. Performances of these methods are hampered by some limitations 
which include huge computational time consumption. So in this work, a 3-stage determination of damage location using the Mamdani-Adaptive Genetic-Sugeno model has been proposed. The work also tries to compare the performances of individual methods, and the results are described in tables.

From Tables 1-3 the results of the individual methods are given respectively. These tables compare the results from these methods with those from the finite element analysis. Tables 4 and 5 give the comparison of the results from the 3-stage Mamdani-Adaptive Genetic-Sugeno model with finite element analysis and experimental analysis respectively. For the validation of the results from the Mamdani-Adaptive Genetic-Sugeno model, they are compared with the results from the experimental analysis. Two sets of data are used for two different comparisons due to the availability of different data sets from the two analyses. The performance comparison is done in terms of the percentage error. The percentage error has been determined using equations (7.1).

The following table shows the results of the proposed technique (3-stage Mamdani-Adaptive Genetic-Sugeno model).

Table 1. Comparison of the results of FLS (Mamdani FIS) with FEA for a cantilever beam

\begin{tabular}{|c|c|c|c|c|c|c|c|c|c|c|}
\hline No. & $\begin{array}{c}r f f \\
\text { from }\end{array}$ & $\begin{array}{c}r s f \\
\text { from }\end{array}$ & $\begin{array}{c}r t f \\
\text { from }\end{array}$ & $\begin{array}{c}r c d \\
\text { from }\end{array}$ & $\begin{array}{c}r c l \\
\text { from }\end{array}$ & $\begin{array}{c}\text { rcd using } \\
\text { the FLS }\end{array}$ & $\begin{array}{c}\text { rcl using } \\
\text { the FLS } \\
\text { tech }\end{array}$ & $\begin{array}{c}\text { percent. } \\
\text { error } \\
r c d\end{array}$ & $\begin{array}{c}\text { percent } \\
\text { error } \\
r c l\end{array}$ & $\begin{array}{c}\text { Total } \\
\text { error }\end{array}$ \\
\hline \hline 1 & 0.9923 & 0.9912 & 0.9966 & 0.325 & 0.21875 & 0.307148 & 0.206701 & 5.493 & 5.508 & 5.5005 \\
\hline 2 & 0.9931 & 0.9926 & 0.9978 & 0.300 & 0.20625 & 0.283536 & 0.194884 & 5.488 & 5.511 & 5.4995 \\
\hline 3 & 0.9946 & 0.9942 & 0.9972 & 0.2875 & 0.23125 & 0.271607 & 0.218550 & 5.528 & 5.492 & 5.5100 \\
\hline 4 & 0.9959 & 0.99772 & 0.9990 & 0.125 & 0.21875 & 0.118106 & 0.206728 & 5.515 & 5.496 & 5.5055 \\
\hline 5 & 0.9974 & 0.9977 & 0.9965 & 0.275 & 0.36250 & 0.259867 & 0.342505 & 5.503 & 5.516 & 5.5095 \\
\hline
\end{tabular}

Table 2. Comparison of the results of FLS (Sugeno FIS) with FEA for a cantilever beam

\begin{tabular}{|c|c|c|c|c|c|c|c|c|c|c|}
\hline No. & $\begin{array}{c}r f f \text { from } \\
\text { exp. } \\
\text { analysis }\end{array}$ & $\begin{array}{c}r s f \text { from } \\
\text { exp. } \\
\text { analysis }\end{array}$ & $\begin{array}{c}r t f \text { from } \\
\text { exp. } \\
\text { nalysis }\end{array}$ & $\begin{array}{c}r c d \text { from } \\
\text { exp. } \\
\text { analysis }\end{array}$ & $\begin{array}{c}r c l \text { from } \\
\text { exp. } \\
\text { analysis }\end{array}$ & $\begin{array}{c}\text { rcd using } \\
\text { the FLS } \\
\text { techique }\end{array}$ & $\begin{array}{c}\text { rcl using } \\
\text { the FLS } \\
\text { technique }\end{array}$ & $\begin{array}{c}\text { percent. } \\
\text { error } \\
r c d\end{array}$ & $\begin{array}{c}\text { percent, } \\
\text { error } \\
r c l\end{array}$ & $\begin{array}{c}\text { Total } \\
\text { error }\end{array}$ \\
\hline \hline 1 & 0.9923 & 0.9912 & 0.9966 & 0.325 & 0.21875 & 0.307795 & 0.207152 & 5.294 & 5.302 & 5.2980 \\
\hline 2 & 0.9931 & 0.9926 & 0.9978 & 0.300 & 0.20625 & 0.284136 & 0.195321 & 5.288 & 5.299 & 5.2935 \\
\hline 3 & 0.9946 & 0.9942 & 0.9972 & 0.2875 & 0.23125 & 0.272211 & 0.219008 & 5.318 & 5.294 & 5.3060 \\
\hline 4 & 0.9959 & 0.99772 & 0.9990 & 0.125 & 0.21875 & 0.118373 & 0.207123 & 5.302 & 5.315 & 5.3085 \\
\hline 5 & 0.9974 & 0.9977 & 0.9965 & 0.275 & 0.36250 & 0.260403 & 0.343284 & 5.308 & 5.301 & 5.3045 \\
\hline
\end{tabular}

Table 3. Comparison of the results of Adaptive Genetic Algorithm (AGA) with FEA for a cantilever beam

\begin{tabular}{|c|c|c|c|c|c|c|c|c|c|c|}
\hline No. & $\begin{array}{l}\text { rff } \\
\text { from } \\
\text { FEA }\end{array}$ & $\begin{array}{l}r s f \\
\text { from } \\
\text { FEA }\end{array}$ & $\begin{array}{l}\text { rtf } \\
\text { from } \\
\text { FEA }\end{array}$ & $\begin{array}{l}\text { rcd } \\
\text { from } \\
\text { FEA }\end{array}$ & $\begin{array}{l}r c l \\
\text { from } \\
\text { FEA }\end{array}$ & $\begin{array}{l}\text { rcd using } \\
\text { the AGA } \\
\text { techique }\end{array}$ & $\begin{array}{l}\text { rcl using } \\
\text { the AGA } \\
\text { technique }\end{array}$ & $\begin{array}{c}\text { percent } \\
\text { error } \\
\text { rcd }\end{array}$ & $\begin{array}{c}\text { percent. } \\
\text { error } \\
r c l\end{array}$ & $\begin{array}{l}\text { Total } \\
\text { error }\end{array}$ \\
\hline 1 & $\overline{0.9923}$ & 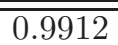 & 0.9966 & $\overline{0.325}$ & 0.21875 & $\overline{\overline{0.308016}}$ & $\overline{0.207342}$ & 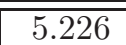 & 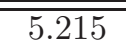 & 5.2205 \\
\hline 2 & 0.9931 & 0.9926 & 0.9978 & 0.300 & 0.20625 & 0.284406 & 0.195535 & 5.198 & 5.195 & 5.1965 \\
\hline 3 & 0.9946 & 0.9942 & 0.9972 & 0.2875 & 0.23125 & 0.272585 & 0.219207 & 5.188 & 5.208 & 5.1980 \\
\hline 4 & 0.9959 & 0.99772 & 0.9990 & 0.125 & 0.21875 & 0.118520 & 0.207351 & 5.184 & 5.211 & 5.1975 \\
\hline 5 & 0.9974 & 0.9977 & 0.9965 & 0.275 & 0.36250 & 0.260678 & 0.343690 & 5.208 & 5.189 & 5.1985 \\
\hline
\end{tabular}


Table 4. Comparison of the results of MAS with FEA for a cantilever beam

\begin{tabular}{|c|c|c|c|c|c|c|c|c|c|c|}
\hline No. & $\begin{array}{c}r f f \\
\text { from } \\
\text { FEA }\end{array}$ & $\begin{array}{c}\text { fsf } \\
\text { from }\end{array}$ & $\begin{array}{c}\text { FEA } \\
\text { from }\end{array}$ & $\begin{array}{c}\text { FEd } \\
\text { from }\end{array}$ & $\begin{array}{c}\text { rcl } \\
\text { from } \\
\text { FEA }\end{array}$ & $\begin{array}{c}\text { rcd using } \\
\text { the MAS }\end{array}$ & $\begin{array}{c}\text { rcl using } \\
\text { the MAS } \\
\text { thechiqu }\end{array}$ & $\begin{array}{c}\text { percent. } \\
\text { technique }\end{array}$ & $\begin{array}{c}\text { percent. } \\
\text { eror } \\
\text { error } \\
\text { rcl }\end{array}$ & $\begin{array}{c}\text { Total } \\
\text { error }\end{array}$ \\
\hline \hline 1 & 0.9923 & 0.9912 & 0.9966 & 0.325 & 0.21875 & 0.309969 & 0.208659 & 4.625 & 4.613 & 4.619 \\
\hline 2 & 0.9931 & 0.9926 & 0.9978 & 0.300 & 0.20625 & 0.286233 & 0.196756 & 4.589 & 4.603 & 4.596 \\
\hline 3 & 0.9946 & 0.9942 & 0.9972 & 0.2875 & 0.23125 & 0.274261 & 0.220643 & 4.605 & 4.587 & 4.596 \\
\hline 4 & 0.9959 & 0.9977 & 0.9990 & 0.125 & 0.21875 & 0.119255 & 0.208655 & 4.596 & 4.615 & 4.6055 \\
\hline 5 & 0.9974 & 0.9977 & 0.9965 & 0.275 & 0.36250 & 0.262259 & 0.345865 & 4.633 & 4.589 & 4.611 \\
\hline
\end{tabular}

Table 5. Comparison of the results of MAS with experimental analysis for a cantilever beam

\begin{tabular}{|c|c|c|c|c|c|c|c|c|c|c|}
\hline No. & $\begin{array}{c}r f f \text { from } \\
\text { exp. } \\
\text { analysis }\end{array}$ & $\begin{array}{c}\text { rsf from } \\
\text { exp. } \\
\text { analysis }\end{array}$ & $\begin{array}{c}r t f \text { from } \\
\text { exp. } \\
\text { analysis }\end{array}$ & $\begin{array}{c}r c d \text { from } \\
\text { exp. } \\
\text { analysis }\end{array}$ & $\begin{array}{c}\text { rcl from } \\
\text { exp. } \\
\text { analysis }\end{array}$ & $\begin{array}{c}\text { rcd using } \\
\text { the MAS } \\
\text { techique }\end{array}$ & $\begin{array}{c}\text { rcl using } \\
\text { the MAS } \\
\text { technique }\end{array}$ & $\begin{array}{c}\text { percent. } \\
\text { error } \\
\text { rcd }\end{array}$ & $\begin{array}{c}\text { percent } \\
\text { error } \\
r c l\end{array}$ & $\begin{array}{c}\text { Total } \\
\text { error }\end{array}$ \\
\hline \hline 1 & 0.9973 & 0.9914 & 0.9995 & 0.34375 & 0.46875 & 0.327862 & 0.447131 & 4.622 & 4.612 & 4.617 \\
\hline 2 & 0.9974 & 0.9890 & 0.9999 & 0.375 & 0.5 & 0.357795 & 0.47691 & 4.588 & 4.618 & 4.603 \\
\hline 3 & 0.99816 & 0.9982 & 0.9979 & 0.25 & 0.375 & 0.238495 & 0.35778 & 4.602 & 4.592 & 4.597 \\
\hline 4 & 0.9988 & 0.9981 & 0.9989 & 0.21875 & 0.40625 & 0.208659 & 0.387502 & 4.613 & 4.615 & 4.614 \\
\hline 5 & 0.9892 & 0.9996 & 0.9981 & 0.35 & 0.1875 & 0.333862 & 0.178879 & 4.611 & 4.598 & 4.6045 \\
\hline
\end{tabular}

\section{Conclusions}

The conclusions drawn from the proposed work can be depicted as follows.

From Tables 1-4, we can get results of the individual methods as well as results from the proposed Mamdani-Adaptive Genetic-Sugeno model. From the comparison of the results of the three individual methods, it can be observed that the adaptive genetic algorithm gives better results as compared to the other two methods (Mamdani FIS and Sugeno FIS). The average total error for the Mamdani FIS is $5.5031 \%$, for the Sugeno FIS is $5.30175 \%$, for the adaptive genetic algorithm is $5.2022 \%$. After analyzing the average total error values of these three methods, it can be said that the adaptive genetic algorithm performs better than the other two methods. From the two of the methods (Mamdani FIS and Sugeno FIS), the Sugeno FIS performs better than the Mamdani FIS. The Sugeno type FIS was designed in order to achieve higher computational effectiveness as compared to the Mamdani FIS. This is possible as the defuzzification of outputs is avoided. Its advantage also lies in involving the functional dependencies of output variables on input variables.

Tables 4 and 5 give the comparison of the results from the 3-stage Mamdani-Adaptive Genetic-Sugeno model with finite element analysis and experimental analysis respectively. The results from the 3 -stage analysis are compared with the results from the experimental analysis, for validation of the results from the proposed method. The average total error is found to be $4.6055 \%$ and $4.6071 \%$ when compared with the results from the finite element analysis and experimental analysis respectively. It is also noticed that the proposed method gives better results as compared to the individual methods. From the analysis of the results and the method, it can be said that the proposed method and all the individual methods can be treated as a robust tool for structural damage detection. The proposed method can be analyzed using different compatible commercial software packages as a future work so that the best method can be found out, which can handle the time consumption and the human error during calculation.

After a thorough literature survey on damage detection, it has been observed that the Artificial Intelligence (AI) techniques are emerging as a powerful tool for damage detection. Lots of work have been done using standalone methods. From different research works, it has been noticed that hybridized methods give better results as compared to standalone methods. This 
happens because the hybridized methods retain the goodness of their elementary algorithms. The hybridized algorithms are so designed and developed that each component of the algorithm tries to overcome shortcomings of other components.

In the current research work, Fuzzy Logic (FL) and Genetic Algorithm (GA) have been considered. Lots of research work have been carried away using fundamental FL and GA. Many researchers have also provided shortcomings of these methods. So, this work tries to overcome the shortcomings of the fundamental methods. Fuzzy Logic consists of two types of inference engines, and both of them are good but when the results and procedures are compared, T-S FIS gives better results. Genetic Algorithm is one of the unique evolutionary algorithms based on Darwin's theory of natural selection and evolution. One of the foremost shortcoming of the algorithm is that it could not provide a global solution. The algorithm sometimes gets stuck in the local solution. So to get rid of the above stated problem, regression analysis has been added. This data analysis process generates a relation between the input and output variables. Out of the three steps, the T-S FIS gives best results, so it has been put in the final step. The other two methods are also organized according to their efficiency.

\section{References}

1. Chandrashekhar M., Ganguli R., 2009, Structural damage detection using modal curvature and fuzzy logic, Structural Health Monitoring, 8, 4, 267-282

2. Dervilis N., Worden K., Cross E.J., 2015, On robust regression analysis as a means of exploring environmental and operational conditions for SHM data, Journal of Sound and Vibration, 347, 279-296

3. Fegade P.V., Mahajan J.A., Bhole G.P., 2014, Study on vibration analysis of an uncrack and cracked cantilever beam, International Association of Scientific Innovation and Research, 10, 4, 340-343

4. Jaiswal N.G., Pande D.W., 2015, Sensitizing the mode shapes of beam towards damage detection using curvature and wavelet transform, International Journal Of Scientific and Technology Research, 4, 4, 266-272

5. Holland J.H., 1992, Genetic algorithms, Scientific American, 66-72

6. Khaji N., Menrjoo M., 2014, Crack detection in a beam with an arbitrary number of transverse cracks using genetic algorithms, Journal of Mechanical Science and Technology, 28, 3, 823-836

7. Niezrecki C. (EDIt.), 2015, Structural Health Monitoring and Damage Detection, Volume 7, Proceedings of the 33rd IMAC, A Conference and Exposition on Structural Dynamics, ISBN 978-3$-319-15230-1$

8. Parhi D.R., Choudhury S., 2011, Intelligent fault detection of a cracked cantilever beam using fuzzy logic technology with hybrid membership functions, International Journal of Artificial Intelligence and Computational Research, 3, 1, 9-16

9. Pawar P.M., Reddy K.V., Ganguli R., 2007, Damage detection in beams using spatial Fourier analysis and neural networks, Journal of Intelligent Material Systems and Structures, 18, 4, 347-359

10. Peng Z.K., Lang Z.Q., Billings S.A., 2007, Crack detection using nonlinear output frequency response functions, Journal of Sound and Vibration, 301, 3, 777-788

11. Ranjbaran A., Ranjbaran M., 2013, New finite-element formulation for buckling analysis of cracked structures, Journal of Engineering Mechanics, 140, 5

12. Saridakis K.M., Chasalevris A.C., Papadopoulos C.A., Dentsoras A.J., 2008, Applying neural networks, genetic algorithms and fuzzy logic for the identification of cracks in shafts by using coupled response measurements, Computers and Structures, 86, 11, 1318-1338 
13. Shahidi S.G., Nigro M.B., Pakzad S.N., Pan Y., 2015, Structural damage detection and localisation using multivariate regression models and two-sample control statistics, Structure and Infrastructure Engineering, 11, 10

14. Thatoi D., Choudhury S., Jena P.K., 2014, Fault diagnosis of beam-like structure using modified fuzzy technique, Advances in Acoustics and Vibration

15. Vakil-Baghmisheh M.T., Peimani M., Sadeghi M.H., Ettefagh M.M., 2008, Crack detection in beam-like structures using genetic algorithms, Applied Soft Computing, 8, 2, 1150-1160

16. Verma P., Rathore M., Gupta R., 2013, Vibration control of cantilever beam using fuzzy logic controller, International Journal of Science Engineering and Technology Research, 2, 4, 906-909

17. Waghulde K.B., Kumar B., 2014, Vibration analysis of cracked cantilever beam with suitable boundary conditions, International Journal of Innovative Science, Engineering and Technology, 1, $10,20-24$

18. Yuan H., Peng C., Lin Q., Zhang B., 2014, Simulation of tensile cracking in earth structures with an adaptive RPIM-FEM coupled method, KSCE Journal of Civil Engineering, 18, 7, 2007-2018

19. ZHU F., WU Y., 2014, A rapid structural damage detection method using integrated ANFIS and interval modeling technique, Applied Soft Computing, 25, 473-484

Manuscript received April 7, 201\%; accepted for print June 21, 2017 